\title{
ON SOME PECULIAR FEATURES IN THE SEQUENCES OF THE OLD OPEN STAR CLUSTERS
}

\author{
A. MAEDER \\ Geneva Observatory, Switzerland
}

\begin{abstract}
In spite of the rather good agreement between the theory of stellar evolution and the observations, there exist some difficulties when one compares closely the sequences of open star clusters and the theoretical isochrones. Several, if not all, of the old open star clusters seem to be concerned, especially those which are accurately measured, namely Praesepe, NGC 2360, 752, 3680 and M67. The problem concerns the gap occuring in the HR diagram at the end of the phase of hydrogen burning in the core; it corresponds to the phase of hydrogen exhaustion (or of overall contraction). The sequence of M67 has been studied by Racine (1971) and TorresPeimbert (1971). The well apparent gap is located farther from the zero-age main sequence than indicated by the models and the hook towards a larger $T_{\text {eff }}$ predicted during this phase is not observed. Differences in chemical composition may not be held responsible for these anomalies. From Torres-Peimbert's models, it may be assumed that neither solar type, nor super metal rich composition are able to reduce the discrepancies. As a further illustration, let us mention the case of NGC 752. In Table I, the main features related to the gap are examined: the disagreement, like in M67, essentially concern features 1 and 2. The observations are based on a recent
\end{abstract}

TABLE I

\begin{tabular}{lcccc}
\multicolumn{5}{c}{ NGC $752:$ features of the sequence } \\
Features & Observed & $\begin{array}{l}\text { Observed on } \\
\text { Bell's fig. }\end{array}$ & $\begin{array}{l}\text { Iben (1967a) } \\
\text { log age }=9.2\end{array}$ & $\begin{array}{l}\text { Hejlesen } \text { et al. } \\
\text { log age }=9.2\end{array}$ \\
\hline 1 & 1.75 & 1.60 & 0.75 & 0.85 \\
2 & 0.30 & 0.35 & 0.30 & 0.30 \\
3 & -0.02 & 0.00 & 0.11 & 0.10 \\
4 & 0.20 & 0.15 & 0.50 & 0.30 \\
\hline
\end{tabular}

\footnotetext{
1 height (in magnitude) of the top of the gap above the main sequence.

2 vertical size (in magnitude) of the gap.

3 horizontal size (in $B-V$ index) of the gap. (Top minus base.)

4 height (in magnitude) of the subgiant peak above the top of the gap.
}

study of Grenon and Mermillod (1973) and on Bell's data (1972). Bell has also mentioned the existence of discrepancies. As in M67, the gap is too far from the zero-age main sequence and does not present any sudden turning towards a larger $T_{\text {eff }}$.

It is well established (Iben, 1967b) that the presence of the gap depends in a oneto-one fashion with the occurence of convective mixing during the phase of hydrogen burning in the core. So, it is possible that the above anomalies are an indication of 
some modifications of convection in the stellar interiors relatively to what is generally assumed in computations of stellar models. In order to make some check on these points, we have tested the effects of overshooting of the convective core on the evolutionary tracks. In the present calculations, we have introduced this effect according to the discussion of Dilke and Gough (1972) about the solar neutrino problem, by limiting the ratio $H_{X} / H_{p}$ to some critical value $\zeta . H_{X}$ and $H_{p}$ are the scale heights of the hydrogen content $X$ and of pressure $p$. In fact, other hydrodynamical processes may produce the overshooting of the convective core. Effects of this kind have been discussed for example by Spiegel (1972), Shaviv and Salpeter (1973). A common characteristic of these processes is that they are all producing a smoothing of the $\mu$-gradient during evolution. Some results are shown in Figure 1. The smoothing of the $\mu$-gradients tends to reduce the observational discrepancies. The gap occurs farther from the zero-age main sequence and does not present the large hook to the left. The case $\zeta=0.5$, which lies close to the observations, corresponds to a supplementary smoothing of the $\mu$-gradient affecting a region of $6 \%$ of the total stellar mass above the con-

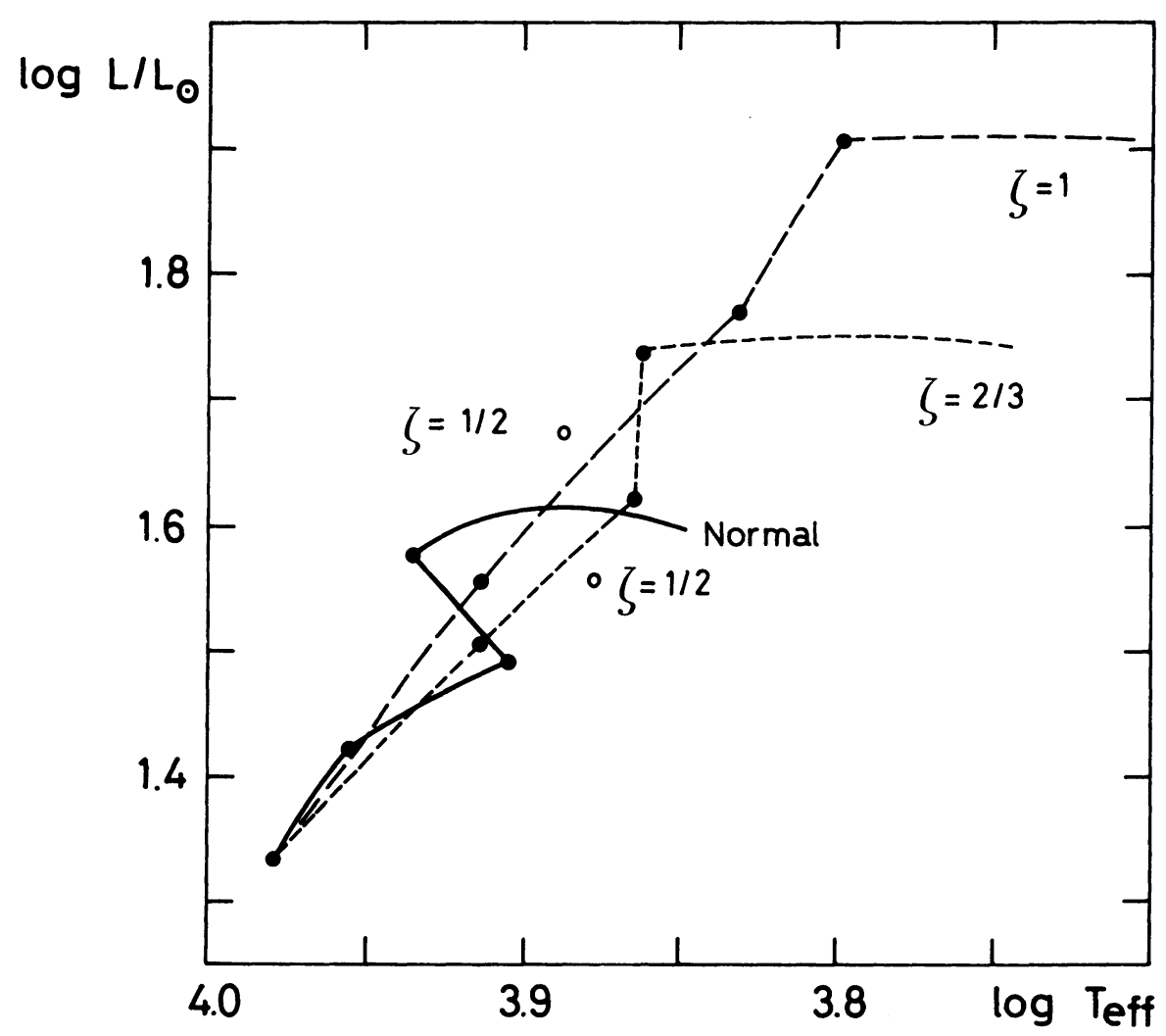

Fig. 1. Evolutionary tracks in the HR diagram for various cases of smoothing of the $\mu$-gradients in a $2.25 M_{\odot}$ star. The track labelled 'normal' represents evolution computed under usual assumptions. 
vective core. As more nuclear fuel is available during the phase of hydrogen burning in the core, such an effect leads to an increase of $40 \%$ in the duration of this phase.

\section{References}

Bell, R. A.: 1972, Monthly Notices Roy. Astron. Soc. 157, 147.

Dilke, F. W. W. and Gough, D. O.: 1972, Nature 240, 262.

Grenon, M. and Mermillod, J. C.: private communication.

Hejlesen, P. M., Jorgensen, H. E., Petersen, J. O., and Romcke, L. : 1972, in G. Cayrel de Strobel and A. M. Delplace (eds.), 'Stellar Ages', IAU Colloq. 17, XVII-1.

Iben, I.: 1967a, Astrophys. J. 147, 624.

Iben, I.: 1967b, Ann. Rev. Astron. Astrophys. 5, 571.

Racine, R.: 1971, Astrophys. J. 168, 393.

Shaviv, G. and Salpeter, E. E. : 1973, Astrophys. J. 184, 191.

Spiegel, E. A.: 1972, Ann. Rev. Astron. Astrophys. 10, 261.

Torres-Peimbert, S.: 1971, Bol. Obs. Tonantzintla Tacubaya 6, 3.

\section{DISCUSSION}

Demarque: I was very interested in this star because Prather and I have been making very similar calculations where we've been worried about a discrepancy with observation for a long time. We have done it in a much more simple minded way, nothing as sophisticated as what you have done, and just looked into the effects of some slight amount of overshooting in a convective core. Of course you do get the same sort of effect, and in fact you find that if you do it this way that it is restricted to a region of the HR diagram which is more or less where M67 is. I would warn that the model of Dilke and Gough is controversial and I wouldn't want to tie my theory to their paper.

Maeder: Yes, I agree. My aim is mainly to show that a smoothing of the $\mu$-gradient results in a modification of the evolutionary tracks in the sense required by the observations.

Iben: This is also going to have a tremendous influence on the position of core helium burning stars, in particular it will affect the mass-luminosity relationship. Have you carried the analysis in this direction?

Maeder: No, but the evolution of the red giant stars will probably be also affected by this criterion, because the internal history of the star is different when we come to the red giant region, but I did not look at this. 